\title{
DOENÇA DO TRATO URINÁRIO INFERIOR DOS FELINOS: ABORDAGEM SOBRE CISTITE IDIOPÁTICA E UROLÍTIASE EM GATOS
}

\author{
Michele Ferreira de Assis' ${ }^{1}$ Marilda Onghero Taffarel${ }^{2}$ \\ 'Mestranda, Universidade Estadual de Maringá, Campus Umuarama, Paraná, Brasil, \\ e-mail: tchelibianchini@gmail.com \\ ²Doutora, Docente da Universidade Estadual de Maringá, Campus Umuarama, \\ Paraná, Brasil, \\ Recebido em: 06/04/2018 - Aprovado em: 10/06/2018 - Publicado em: 20/06/2018 \\ DOI: 10.18677/EnciBio 2018A36
}

\begin{abstract}
RESUMO
O termo doença do trato urinário inferior dos felinos (DTUIF), é um termo geral para descrever diversas manifestações que causam inflamação na vesícula urinária e uretra, independente da afecção primária. A sintomatologia é bastante semelhante dentre as doenças que acometem esse sistema, portanto, o diagnóstico definitivo só é definido a partir do histórico clínico completo, além de exames laboratoriais e de imagem. Os sintomas mais comuns da doença são hematúria, estrangúria, polaciúria, periúria, disúria, vocalização no momento de urinar entre outros. Diversos distúrbios estão associados as causas de DTUIF, como cistite idiopática felina (CIF), urolitíase, tampãoes uretrais, anormalidades anatômicas, infecções, neoplasias e problemas comportamentais. A afecção também pode ser classificada em obstrutiva ou não obstrutiva. Vários fatores estão relacionados ao risco do animal desenvolver DTUIF. Dentre eles, gatos confinados, que vivem em ambientes fechados, com dieta predominantemente seca, baixo consumo de água, ambientes estressantes, como super populações de gatos, podem contribuir para o aparecimento dos sinais clínicos. O tratamento é direcionado de acordo com o diagnóstico definitivo, mas de maneira geral estão relacionados com o uso de analgésicos no controle da dor, antiinflamatórios não esteroidais, antidepressivos, suplementação com glicosaminoglicanas, uso de feromônios faciais sintéticos, estímulo do aumento do consumo de água, manejo dietético para redução de peso, manejo dietético para prevenção ou diluição de urólitos, enriquecimento ambiental, com modificação do ambiente e consequentemente diminuição do estresse.
\end{abstract}

PALAVRAS-CHAVE: bexiga, cálculo ; Sindrome de pandora.

\section{DISEASE OF LOWER URINARY TRACT OF FELINES: APPROACH ON IDIOPATHIC CYSTITIS AND UROLITHITIS IN CATS - LITERATURE REVIEW}

\begin{abstract}
:
The term feline lower urinary tract disease (FLUTD) is a general term for describing several manifestations that cause inflammation in the urinary vesicle and urethra, regardless of the primary condition. The Symptomatology is very similar among the diseases that affect this system, therefore, the definitive diagnosis is only defined from the complete clinical history, in addition to laboratory and imaging tests. The
\end{abstract}


most common symptoms of the disease are hemorrhage, strangury, polaciuria, periury, dysuria, vocalization at the moment of urinating among others. Several disorders are associated with the causes of FLUTD, such as feline idiopathic cystitis (FIC), urolithiasis, urethral buffers, anatomical abnormalities, infections, neoplasms and behavioral problems. The condition can also be classified as obstructive or nonobstructive. Several factors are related to the animal's risk of developing FLUTD. Among them, confined animals, living indoors, with a predominantly dry diet, low water consumption, and stressful environments, such as super populations of cats, may contribute to the appearance of clinical signs. The treatment is directed according to the definitive diagnosis, but in general they are related to the use of analgesics in pain control, non-steroidal anti-inflammatory drugs, antidepressants, glycosaminoglycan supplementation, use of synthetic pheromones, stimulation of increased consumption of water, dietary management for weight reduction, dietary management for prevention or dilution of uroliths, environmental enrichment, with modification of the environment and consequently decrease of stress.

KEYWORDS: Pandora syndrome, bladder, stone

\section{INTRODUÇÃO}

A doença do trato inferior dos felinos (DTUIF) ainda é um dos problemas mais frequentes que resultam em padrões alterados da micção (DULANEY et al., 2017). O termo DTUIF é usado para descrever um conjunto de afecções que causam inflamação da vesícula urinária e lou uretrais e que podem acarretar inclusive perda aguda da função renal (JUNIOR; 2015; NERI, 2016). Qualquer afecção do trato urinário inferior dos felinos irá causar sinais clínicos como: hematúria, polaciúria, estrangúria, micção fora da caixa de areia, obstrução uretral ou não, vocalização no momento da micção entre outros. Tais manifestações são evidenciadas isoladas ou em conjunto (FERREIRA, 2014; ROBERTSON, 2014). Como as sintomatologias são muito similares, independente de qual for a etiologia primária, são necessários exames laboratoriais e de imagem para a confirmação do diagnóstico (JUNIOR; 2015; DORSCH, 2016).

Diversos distúrbios são considerados como causa primária da DTUIF, como Infecção do trato urinário (ITU), plugs uretrais, urólitos, malformações, neoplasias, distúrbios de comportamento relacionadas ao estresse e quando a causa não é identificada a afecção é considerada idiopática, ou cistite idiopática (CIF) (BUFINGTON, 2014; JUNIOR, 2015). Síndrome de Pandora é uma terminologia mais atual utilizada para se referir a DTUIF e CIF, pois existem fatores externos interligados às manifestações clínicas e a afecção muitas vezes não envolve apenas um órgão (BUFINGTON, 2014).

A causa mais comum da doença do trato urinário inferior dos felinos é a cistite idiopática (com 55 a 65\%) dos casos, seguida da urolitíase (15 a 20\%), anormalidades anatômicas e problemas de comportamento (com cerca de 10\%) dos relatos. As etiologias menos comuns são neoplasias (1 a $2 \%$ ) e infecções do trato urinário (que totalizam de 1 a 8\%) (LITTLE, 2016).

Variados fatores estão relacionados aos riscos dos felinos desenvolverem a DTUIF. Pacientes que vivem em ambientes confinados ou em super populações, gatos com histórico de obesidade, castrados, com dieta exclusivamente seca e expostos a diversos fatores que favoreçam o estresse, são animais com maior predisposição a desenvolverem os sinais da doença (BUFINGTON, 2014; LITTLE 2016). 
O objetivo deste trabalho de revisão foi de fazer um apanhado das duas afecções do trato urinário inferior dos felinos que mais ocasionam os tutores a levarem seus gatos ao veterinário. Apontar suas possíveis causas, diagnósticos e formas de tratamento, a fim de melhorar a qualidade de vida do paciente felino.

\section{CISTITE IDIOPÁTICA FELINA (CIF)}

A cistite idiopática felina ou também conhecida como cistite intersticial, é um processo inflamatório que intriga os pesquisadores, pois sua fisiopatologia ainda não foi compreendida totalmente. Acredita-se que existem várias etiologias envolvidas, que podem estar ou não relacionadas com a síndrome. Estima-se que essa afecção afeta de 250.000 a 500.000 gatos todos os anos nos Estados Unidos da América (BRADLEY, 2014). As recidivas são comuns, o que pode frustrar tanto o tutor, quanto o clínico. Podendo apresentar-se de forma aguda ou crônica, obstrutiva ou não obstrutiva (BUFFINGTON, 2011; LITTLE, 2016). Muitas vezes apresenta caráter auto limitante, pois segundo os autores, quando não obstrutiva, os sinais clínicos de disúria, polaciúria, periúria e hematúria duram em média de um a sete dias e posteriormente pode ocorrer completa resolução da sintomatologia (KRUGER et al., 2015). É por muitas vezes comparada à cistite intersticial em humanos e os sinais clínicos podem ser acentuados devido ao estresse (HAUSER, 2015).

Em um estudo realizado por Defauw et al. (2011), foram avaliados 64 gatos com sinais de CIF em relação a possíveis fatores de risco associados a doença, como número de gatos por casa, número de caixas de areia por animal, ambiente enriquecido ou não, mudança de casa, introdução de um novo animal na casa ou de um bebê, atrito com outro ou outros gatos, local onde o animal dorme, entre outros. Este estudo evidenciou que gatos que vivem em ambientes fechados têm mais ganho de peso, menos comportamento de caça, baixa ingestão de água e são de modo geral mais lentos e mais nervosos em comparação com animais que tem acesso ao exterior da residência. Além disso evidenciou que não existe predisposição racial ou diferenças entre animais de pelo longo ou curto para o desenvolvimento da CIF. Em um outro estudo realizado nos Estados Unidos da América por Lekcharoensuk et al. (2001), foi evidenciado que sim, existe predisposição racial para o desenvolvimento da cistite intersticial, sendo as raças mais acometidas: os Persas, Manx e Himaláia e a menos afetada, a raça Siamesa. Entretanto Jones et al. (2011), avaliaram os possíveis fatores de risco associados a doença do trato urinário inferior dos felinos em 189 gatos na Nova Zelândia. Estes concluíram em seu estudo que as raças puras mais acometidas foram os Persas e os Siameses.

Heidenberger (1997), afirma que a maioria dos estudos sobre CIF apontam que os machos de dois a sete anos, com excesso de peso apresentam maiores riscos de apresentarem tal afecção. Buffington et al. (2006) atestam que é cada vez mais claro que o ambiente onde o felino vive e as condições que ele oferece ao mesmo, podem afetar de forma significativa o aparecimento de sinais de CIF. De acordo com Jones et al. (2011), o estresse causado por mudanças frequentes e períodos longos de confinamento, em detrimento de invernos prolongados e chuvosos, além do maior consumo de ração seca e baixo consumo de água também contribuem para o surgimento dos sinais da cistite idiopática felina.

\section{DIAGNÓSTICO}

O diagnóstico é realizado por exclusão, pois não há um teste específico para o diagnóstico da CIF. As queixas dos tutores em relação aos sinais clínicos são 
basicamente os mesmos, como hematúria, estrangúria, polaciúria, disúria. Portanto a realização de histórico completo, incluindo histórico ambiental e alimentar, exame físico, urinálise e exames de imagem como a ultrassonografia abdominal e radiografia se fazem importantes na tentativa de elucidação do diagnóstico (FORRESTER, 2015; LITTLE, 2016).

A urinálise de um gato com DTUIF pode conter várias anormalidades, mas nenhuma destas é conclusiva de CIF. A hematúria é um achado frequente, porém a mesma pode ser induzida pela coleta (cistocentese, cateterismo vesical). Também pode ser evidenciado o aumento de proteína, devido a inflamação neurogênica da mucosa da bexiga, que levará a um extravasamento de proteína do plasma e posteriormente aumento do $\mathrm{pH}$ urinário. Consequentemente esse fato induz a formação de cristais de estruvita. A cultura da urina deverá ser recomendada em gatos com idade acima de 10 anos, ou com animais que foram submetidos a uretrostomia perineal, cateter uretral, animais com densidade urinária baixa ou que apresentam histórico de apresentarem recentes sinais clínicos (LITTLE, 2016).

Os exames de imagem também se fazem necessários. A radiografia é empregada na suspeita de urolitíase e a ultrassonografia para avaliação do lúmen da bexiga, coágulos, massas, cálculos císticos etc. Estima-se que 15 a $20 \%$ dos gatos com sintomas de doença do trato urinário, apresentam cálculos urinários. A cistografia contrastada também pode ser utilizada, principalmente para detectar cálculos radiotransparentes, massas neoplásicas, suspeitas de ruptura de bexiga, anormalidades congênitas, divertículos vesicais etc (FORRESTER 2015; LITTLE, 2016).

\section{TRATAMENTO}

\section{Manejo Ambiental}

Por ser uma doença que pode ter frequentes recidivas, a cistite idiopática geralmente se torna uma afecção frustrante para veterinários e tutores. Segundo estudos, existe a possibilidade de envolvimento do sistema nervoso simpático em situação de estresse, o que estimula a resposta inflamatória criando um processo crônico (BUFFINGTON, 2006).

A bexiga é inervada por neurônios que são constituídos de fibras amielinizadas, que são chamadas de fibras C. Essas, quando ativadas, por vezes levam à liberação de neuropeptídios que causam dor intrapélvica, aumento da permeabilidade vascular da parede da bexiga, contração da musculatura lisa, edema da camada submucosa e degranulação de mastócitos. Dessa forma, são liberados vários mediadores da inflamação, como a histamina, heparina, serotonina, citocinas e prostaglandinas. A liberação dessas substâncias pode intensificar os efeitos das fibras $\mathrm{C}$ e com isso o surgimento de petéquias na região subepitelial da bexiga. Esse sinal pode ser evidenciado em pacientes humanos, mas também em felinos (HOSTUTLER, 2005).

Dessa forma, o tratamento se volta no direcionamento da identificação e redução dos fatores que geram estresse e é essencial que os Tutores tenham suporte e informações a respeito do assunto (WESTTROPPE, 2004). Em relação ao enriquecimento ambiental, é recomendado que o tutor ofereça ao gato uma atmosfera que seja mais apropriada para a espécie, onde ele possa ter o senso do controle sobre o ambiente, como simular o comportamento de caça, escondendo alimentos em brinquedos, estimular o consumo de água, com fontes ou água diretamente na torneira. Além disso, a interação do tutor com o gato é muito 
importante, através de brincadeiras ou do simples afago no pelo (BUFFINGTON, 2002).

Em um estudo realizado por Guy (2014), comprovou que os gatos mantidos no interior das residências preferem caixas de areias com tamanhos maiores do que as comercialmente vendidas. O número de dejetos resultantes da micção dos felinos foi consideravelmente maior do que nas liteiras de tamanhos convencionais, indicando menor retenção urinária pelos animais confinados.

Em relação a alimentação Bell et al. (2015) citam que em um estudo recente, onde 25 gatos com sinais de CIF receberam uma dieta suplementada com ômega 3 e ácidos graxos por um período de 12 meses tiveram uma redução significativa na recidiva dos sinais da doença em relação aos gatos do grupo controle que receberam uma dieta convencional. Em outro experimento Kruger et al. (2015), testaram um alimento com baixos teores de magnésio, acidificante urinário e ácidos graxos ômega-3 enriquecidos com antioxidantes. Este conseguiu reduzir a taxa recorrente em dias de sinais como a hematúria, disúria e estrangúria, porém os efeitos das taxas de recorrência da polaciúria e periúria não foram aparentes, indicando que novos estudos precisam ser realizados. A tabela abaixo mostra uma lista de verificações de recursos que ajudam os tutores a identificar e corrigir possíveis problemas relacionados ao manejo das caixas de areia, água e comida.

TABELA 1 - Lista de verificação de recursos para tutores de gatos em relação ao ambiente, água e comida.

\begin{tabular}{|c|c|c|}
\hline \multirow{2}{*}{$\begin{array}{l}\text { MANEJO DA CAIXA DE AREIA } \\
\text { As caixas de areia são dispostas em mais de um andar em casas } \\
\text { com vários andares. }\end{array}$} & 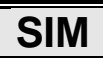 & NÃO \\
\hline & & \\
\hline $\begin{array}{l}\text { As caixas de areia são dispostas de modo a outro animal não } \\
\text { conseguir bisbilhotar enquanto o outro gato usa. }\end{array}$ & & \\
\hline $\begin{array}{l}\text { As caixas de areia são dispostas longe de aparelhos ou ductos de } \\
\text { ar que possam ser ligados inesperadamente enquanto o gato usa. }\end{array}$ & & \\
\hline $\begin{array}{l}\text { A caixa de areia é mantida limpa, com os resíduos retirados o mais } \\
\text { rapidamente possível após o uso (no mínimo diariamente). }\end{array}$ & & \\
\hline $\begin{array}{l}\text { As caixas de areia são lavadas regularmente (no mínimo uma vez } \\
\text { por semana) com detergente suave (ex. sabão para lavagem de } \\
\text { pratos), em vez de limpadores com odor forte. }\end{array}$ & & \\
\hline Material (tipo da areia sanitária) sem odor para a caixa de areia. & & \\
\hline $\begin{array}{l}\text { A marca ou o tipo do material da caixa de areia comercializado são } \\
\text { trocados com pouca frequência (menos de uma vez por mês). }\end{array}$ & & \\
\hline $\begin{array}{l}\text { Se um tipo de areia higiênica é oferecido, coloca-se ele em uma } \\
\text { caixa separada de modo que o gato possa escolher usá-lo se } \\
\text { assim desejar. }\end{array}$ & & \\
\hline $\begin{array}{l}\text { Cada gato tem sua própria caixa de areia em uma localização } \\
\text { conveniente e bem ventilada de modo que ainda confira alguma } \\
\text { privacidade ao gato enquanto a usa. }\end{array}$ & & \\
\hline ALIMENTO E ÁGUA & & \\
\hline $\begin{array}{l}\text { Cada gato sem sua própria tigela de água e alimento em } \\
\text { localização conveniente que proporciona alguma privacidade } \\
\text { enquanto se alimenta ou bebe água. }\end{array}$ & & \\
\hline $\begin{array}{l}\text { Os recipientes são localizados de modo que outro animal não } \\
\text { consiga pular sobre o gato enquanto este se alimenta. }\end{array}$ & & \\
\hline
\end{tabular}


Os recipientes são localizados afastados de aparelhos ou ductos de ar que possam ser ligados inesperadamente enquanto o gato come ou toma água.

Os alimentos são mantidos frescos (diariamente).

Os recipientes são lavados regularmente (no mínimo semanalmente) com detergente suave.

A marca ou o tipo de alimento comercializado é trocado com pouca frequência (menos que mensalmente).

Se uma nova ração for oferecida, coloca-se em um recipiente separado próximo do conhecido, de modo que o gato possa escolher comê-la se assim desejar.

\section{CONSIDERAÇÕES AMBIENTAIS}

Postes para arranhadura são fornecidos.

Brinquedos são fornecidos, oferecidos em rodízio ou substituídos regularmente.

Cada gato tem a oportunidade de se movimentar de uma área mais aquecida ou mais resfriada, caso queira.

Cada gato tem seu próprio espaço de modo que pode utiliza-lo, caso queira.

\section{REPOUSO}

Cada gato tem sua própria área de repouso em localização conveniente que ainda proporcione alguma privacidade.

As áreas de repouso estão localizadas de modo que outro animal não consiga pular sobre o gato enquanto ele repousa.

As áreas de repouso localizam-se afastadas de aparelhos ou ductos de ar que possam ser ligados inesperadamente enquanto o gato descansa.

Se uma nova cama for oferecida, ela é colocada próximo da cama familiar de modo que o gato possa escolher usá-la, se assim o desejar.

Movimento - cada gato tem a oportunidade de se movimentar livremente, explorar, escalar, se espreguiçar, e brincar, caso queira.

Contato social - cada gato tem a oportunidade de se envolver em brincadeiras com outros animais, ou com o tutor, caso queira.

Fonte: Adaptado do livro O gato, medicina interna, (LITTLE, 2016).

\section{TRATAMENTO}

\section{Terapia Medicamentosa}

Nos casos em que a CIF se torna frequente e consequentemente crônica, sendo refratária a melhorias como o enriquecimento ambiental (arranhadores, brinquedos), manejo adequado das caixas de areia, potes de comida maiores e múltiplos, estímulo a caça e ao aumento do consumo de água, maior interatividade do tutor com o felino e etc. Quando essas medidas são tomadas, mas ainda se tem casos refratários, muitas vezes se faz necessário o uso da terapia medicamentosa, como o caso dos antidepressivos tricíclicos (amtripitilina) e de analgésicos como a 
buprenorfina, butorfanol, fentalina e os antiinflamatórios não esterodais (ROBERTSON, 2014; LITTLE, 2016).

A amitriptilina é um antidepressivo tricíclico com propriedades anticolinérgicas, anti-histamínicas, simpaticolíticas, anti-inflamatórias e analgésicas. É comumente utilizada no tratamento da cistite intersticial refratária em felinos e diversos distúrbios de ansiedade e separação em cães. Nos humanos também é administrada para controle dos sinais da cistite idiopática e no tratamento da dor neuropática crônica (LITTLE, 2016; FENELON, 2017).

Os seus benefícios são vistos a longo prazo e a dose varia de 2,5 a 12,5 mg, por via oral, a cada 24 horas. A dosagem deve ser gradativamente aumentada para mais, de acordo com a melhora da sintomatologia e quando não houver resposta na redução dos sinais clínicos em até quatro meses, a mesma deve ser paulatinamente descontinuada (HOSTUTLER, 2005). Em um estudo multicêntrico realizado na Holanda por Kraijer et al. (2003), testaram os efeitos da amitriptilina na dose de 10 $\mathrm{mg}$, por via oral, a cada 24 horas, durante sete dias em gatos com sinais clássicos de DTUIF que já haviam apresentado os mesmos sinais anteriormente. Este concluíram que os resultados obtidos não suportam a hipótese de que a amitriptilina usada por esse período traga benefícios para diminuição ou resolução dos sinais da DTUIF em felinos.

O estresse durante o transporte, seja por viagens ou mesma uma visita ao veterinário é um fator importante em relação aos felinos, porque muitos deixam de receber maior atenção, o que inclui consultas preventivas ao veterinário, devido nervosismo e ansiedade. Em uma pesquisa recente, a Gabapentina foi administrada em dose única 90 minutos antes dos gatos serem transportados até o veterinário por seu tutor. A conclusão do trabalho foi que houve diminuição significativa do estresse, tanto no transporte, quanto na manipulação durante a consulta (HAAFTEM, 2017). $\mathrm{Na}$ espécie humana a gabapentina também é utilizada para o tratamento de várias desordens de ansiedade (BERLIN, 2015).

\section{Glicosaminoglicanos}

As glicosaminas são uma classe de mucopolissacarídeos que apresentam características hidro-repelentes e o rompimento de sua camada na bexiga expõe o uroepitélio aos agentes tóxicos da urina e uma vez lesado é iniciado um processo inflamatório imunomediado ou neurogênico, com liberação de mediadores químicos que levam a sinais de desconforto, dor, entre outros sinais (CERVIGNI, 2015).

A eficácia da utilização das glicosaminoglicanas não está totalmente comprovada cientificamente, entretanto, sabe-se que elas exercem efeitos analgésicos e anti-inflamatórios no tratamento de gatos com cistite grave (JUNIOR, 2015).

\section{Feromônio Facial Felino}

F3 é a fração sintética do feromônio facial felino que mimetiza a marcação natural que os gatos fazem quando esfregam a face nos objetos e pessoas. A substância foi desenvolvida com o intuito de ajudar no tratamento de problemas de comportamento relacionados a ansiedade nos felinos (MOORE et al.; 2004).

Os autores citados a cima, realizaram um estudo piloto na Universidade de Edimburgo, comparando a utilização do spray do feromônio sintético comercial (Faliway® - Ceva) em relação a um spray placebo em gatos com pelo menos seis episódios de CIF nos últimos seis meses. Os resultados indicaram que os felinos que tiveram contato com o spray comercial obtiveram leve melhora nos sinais da 
doença e dessa forma o produto é indicado como parte do tratamento (MOORE et al., 2004).

Em outro experimento Silva (2017), testou o efeito do análogo sintético do feromônio facial felino sobre o nível de cortisol salivar em gatos e evidenciou que em $75 \%$ do animais testados, houve diminuição do nível de cortisol salivar, indicando que esse feromônio pode ajudar no controle do estresse em pacientes felinos com sinais de CIF.

\section{UROLITÍASE}

O trato urinário inferior é um sistema especializado na função de captar, armazenar e liberar a urina de forma cíclica. Este sistema foi arquitetado para produzir uma urina concentrada e eliminar resíduos orgânicos de forma líquida (WAKI ; KOGIKA, 2015).

Determinados resíduos, em especial minerais, quando não eliminados pela urina, podem precipitar e predispor à formação de cristais. Estes, quando retidos no sistema urinário e combinados com outros minerais ou matriz orgânica, podem crescer e formar urólitos (WAKI ; KOGIKA, 2015).

A maioria dos cálculos vesicais encontrados nos felinos é composta por fosfato de amônio e magnésio (estruvita) ou oxalato de cálcio. Outros urólitos, de urato de amônio, cistina, silicato, xantina, fosfato de cálcio, pirofosfato também são encontrados, mas menos relatados (WAKI ; KOGICA, 2015).

A Urolitíase é a segunda causa descrita na literatura que mais causa sinais da doença do trato urinário inferior dos felinos. Os urólitos mais encontrados nos felinos são a estruvita (49\%) e o oxalato de cálcio (41\%), sendo que cálculos formados por outros urólitos somam (10\%) (LITTLE, 2016). As obstruções da uretra devido a estenose causada por cálculos em felinos, chegam a $20 \%$ dos relatos (COOPER, 2015).

\section{Urólitos de Estruvita}

Os cálculos de estruvita ou como também são conhecidos, cálculos de fosfato triplo ou de fosfato amoníaco-magnesiano, na maioria da vezes são formados na bexiga e em urina estéril nos gatos. Porém sua produção pode ser induzida por infecções em gatos com menos de um ano e gatos com mais de 10 anos de vida (WAKI ; KOGICA, 2015; LITTLE, 2016).

A presença de cristais de estruvita na vesícula urinária nem sempre é notada pelos tutores, pois na maioria das vezes, os gatos não apresentam sinais clínicos de DTUIF, com exceção quando ocorre obstrução da uretra em gatos machos. Já os urólitos em contato com a mucosa da bexiga, acarretam em sinais clínicos genéricos do trato urinário inferior (BELL, 2015).

A formação dos urólitos de estruvita são induzidos pelo pH, concentração, e materiais calculogênicos presentes na urina. Tais urólitos podem ser diluídos quando o pH da urina for reduzido abaixo de 6,4 (LEKCHAROENSUK, 2001). Em um estudo realizado por esse mesmo autor, foi evidenciado que gatos alimentados com dietas ricas em fibras, cálcio, fósforo, magnésio, sódio ou potássio aumentam os riscos do desenvolvimento de urólitos de estruvita.

\section{DIAGNÓSTICO}

Frequentemente as radiografias abdominais simples são suficientes para a realização do diagnóstico. Quando os urólitos são muito pequenos ou não 
radiopacos, o diagnóstico pode ser realizado através da cistografia de duplo contraste ou ultrassonografia abdominal (LITTLE, 2016).

\section{TRATAMENTO}

O objetivo do tratamento clínico é a modificação da dieta, dissolução do cálculo e prevenção da formação de novos urólitos. Dietas para gatos com estruvita estéreo, são acidificantes com quantidades reduzidas de magnésio. $O$ tratamento cirúrgico só é indicado quando o paciente apresenta obstrução das vias urinária, e quando os cálculos são refratários a tratamento anteriores (WAKI ; KOGICA, 2015). O estímulo para que ocorra o aumento do consumo de água também faz parte do tratamento (BUFFINGTON, 2002).

\section{Urólitos de Oxalato de Cálcio}

Urólitos de oxalato de cálcio são o segundo tipo de cálculos mais relatado em felinos e são encontrados com maior frequência na pelve renal e nos ureteres de cães e gatos (WAKI \& KOGICA, 2015). Os fatores de risco associados ao seu desenvolvimento estão relacionados com a idade, ou seja, gatos com 7 anos em média tem mais predisposição, assim como as raças: Persa, Himalaio, Birmaneses, English Shorthair, Exotic Shorthair, Havana Brown, Ragdoll, Foreign Shorthair, Scotish Fold, são listadas como mais acometidas. Estudos apontam que na espécie humana os urólitos de oxalato de cálcio, são os mais diagnosticados mundialmente e são mais comumente encontrados em pessoas do sexo masculino. Essa predisposição sexual também é notada na espécie felina (LEKCHAROENSUK, 2000; LITTLE 2016; DAUTON, 2016).

Dietas formuladas para aumentar a acidez urinária podem (como as indicadas para diluição de urólitos de estruvita) elevar os riscos de formação de cálculos de oxalato, assim como dietas com baixos teores de sódio e potássio. Gatos com esses tipos de cálculos apresentam em média uma densidade urinária aumentada (1.040) e pH da urina entre 6,3 a 6,7. Outro fator que pode contribuir para a formação dos cálculos de oxalato é o metabolismo alterado do paciente. Pesquisas demostraram que hipercalcemia mesmo que branda, pode predispor a formação desses urólitos, devido à maior excreção de cálcio na urina e consequentemente ocorre a precipitação de cristais de oxalato. Nos gatos a hipercalcemia é vista em torno de $35 \%$ dos casos. (OSBORN, 1996; BARTGES, 2015).

\section{DIAGNÓSTICO}

Os sinais clínicos como hematúria, polaciúria, estrangúria, periúria são os comumente encontrados em qualquer doença do trato urinário inferior, porém como os cálculos geralmente são múltiplos e pequenos, pode ocorrer obstrução da uretra (WAKI ; KOGICA, 2015; LITTLE, 2016). O diagnóstico para a detecção dos urólitos pode ser por radiografia simples, ou quando pequenos e rádio-opacos pode ser realizada por cistografia de duplo contraste ou ultrassonografia abdominal. Também é importante a realização de exames laboratoriais, como hemograma, bioquímicos, urinálise e cultura da urina (OSBORN, 1996). O diagnóstico definitivo de qualquer urólito é realizado mediante análise química do mesmo (BARTGES, 2015).

\section{TRATAMENTO}

O oxalato de cálcio não é passível de diluição, portanto o objetivo do tratamento clínico é prevenir a sua formação. Porém, na confirmação do diagnóstico 
o tratamento cirúrgico com remoção do/dos cálculos, ou quando possível por hidropropulsão são os métodos mais indicados. Em gatos, mesmo após a retirada dos urólitos existe uma taxa de recidiva de $7,1 \%$ após 23 meses e em cães $50 \%$ em um período de três anos. (WAKI ; KOGICA, 2015).

Para evitar a recorrência dos cálculos, medidas preventivas como aumento da ingesta de água, aumento do consumo de alimentos úmidos, evitar alimentos ricos em oxalato, utilizar rações terapêuticas para o controle da acidiúria mantendo o pH entre 7,0 e 7,5, evitando a formação de cristais. Para promover a reabsorção de cálcio, pode ser feita a suplementação com vitamina B6 e hidroclorotiazida (WAKE; KOGICA, 2015; LITTLE 2016).

\section{Cálculos de Purinas}

Os urólitos de urato de amônio e xantina são pertencentes à família dos cálculos de purinas. O primeiro pode ter ocorrência natural em cães, já os cálculos de xantina que são produtos da conversão das purinas em ácido úrico não têm ocorrência natural nos cães, mas são frequente nos animais dessa espécie submetidos ao tratamento da leishmaniose (MIREAUX, 2014).

Os cálculos de urato apresentam incidência bem menor $(5 \%)$ em relação a estruvita (46\%) e oxalato de cálcio (43\%). A litogênese em gatos ainda não está bem elucidada, mas sabe-se que o aumento da excreção renal e aumento da concentração urinária e sanguínea de ácido úrico, predispõe a formação do cálculo (ALBASAN, 2012). Além disso, outros fatores como: gatos com shunt porto vascular, uma dieta rica em precursores de purina e consequente produção de urina com ph mais ácido e infecções do trato urinário também podem desencadear o problema (GNANANDARAJAH et al.; 2011).

\section{DIAGNÓSTICO E TRATAMENTO}

Como a produção dos cálculos de urato de amônio, podem estar relacionados com alguma disfunção hepática, como o shunt portossistêmico é importante fazer uma avaliação das enzimas hepáticas, ácidos biliares e globulinas nesses animais, mas não quer dizer que obrigatoriamente o paciente com suspeita ou diagnóstico de urolitíase por urato apresenta alguma alteração no órgão em questão (GNANANDARAJAH et al., 2011). Além da avaliação hepática é indicada radiografia abdominal, porém esses cálculos costumam ser pouco radio-opacos, por isso a ultrassonografia e/ou a cistografia contrastada também são indicadas (GNANANDARAJAH et al., 2011).

O tratamento é baseado na correção, caso houver, da alteração hepática e restrições alimentares em relação ao consumo de purinas. Essas proteínas são encontradas em abundância nos frutos do mar, nas carnes, principalmente em vísceras, em grãos como feijão, lentilhas, ervilhas, ovos e seus derivados etc. Em pacientes que apresentam um índice alto de recorrência desses cálculos, pode ser indicado um inibidor da enzima xantina oxidase (alopurinol) na dose de 5 a $10 \mathrm{mg}$, porém devido à grande possibilidade de ocorrerem efeitos adversos, sua indicação é reservada (GNANANDARAJAH et al., 2011).

\section{CONSIDERAÇÕES FINAIS}

A doença do trato urinário inferior dos felinos é uma afecção recorrente, que causa muito sofrimento nos gatos e em seus tutores. O diagnóstico muitas vezes é inconclusivo, mas existem medidas ambientais e medicamentosas para minimizar os efeitos deletérios das várias afecções que afetam esse sistema e proporcionar 
melhor qualidade de vida a esses animais e melhor convivência entre tutores e gatos.

\section{REFERÊNCIAS}

ALBASAN, H.; OSBORNE, C. A.; LULICH, J. P.; LEKCHAOENSUK, C. Risk factors for urate urolithis in cats. Journal of the American Veterinary Medical Association, v.240, n.7, p. 842-847, 2012. Disponível em: <https://avmajournals.avma.org/doi/abs/10.2460/javma.240.7.842 doi: doi.org/10.2460/javma.240.7.842.

BARTGES, J. W.; CALLENS, A. J. Urolithiasis. Veterinary Clinics: Smal Animal Pratice, v.45, n.4, p. 747-768, 2015. Disponível em: <http://www.vetsmall.theclinics.com/article/S0195-5616(15)00046-7/abstract>. doi: doi.org/10.1016/j.cvsm.2015.03.001

BELL, E. T.; LULICH, J. P.; Marked struvite crystalluria and its association with lowerurinary tract signs in a cat with feline idiopathic cystitis. Australian Veterinary Journal, v.93, n. 9, p. 332-335, 2015. Disponível em: <http:///C:/Users/Usuario/Documents/Mestrado/DTUIF/artigos\%20CITADOS\%20NO \%20TEXTO/BELL\%202015.pdf>. doi: 10.1111/avj.12353.

BERLIN, R. R.; BUTLER, P. M.; PERLOFF, M. D. Gabapentin Therapy in Psychiatric Disorders: A Systematic Review. The Primary Care Companion for CNS Disordens, $\quad$ v. 17, $\mathrm{n} \quad 5, \quad 2015 . \quad$ Disponível em: <https://www.ncbi.nlm.nih.gov/pmc/articles/PMC4732322/\#_ffn_sectitle>. doi: 10.4088/PCC.15r01821.

BRADLEY, M. A.; LAPPIN, R. M. Intravesical glycosaminoglycans for obstructive feline idiopathic cystitis: a pilot study. Journal of Feline Medicine and Surgery, v. 16, n. 6, p. 504-506, 2014. Disponível em: http://journals.sagepub.com/doi/abs/10.1177/1098612X13510918. doi: https://doi.org/10.1177/1098612X13510918.

BUFFINGTON, T. C. A. External and internal influences on disease risk in cats. Journal of the American Veterinary Medical Association, v. 220, n. 7, p. 9941002, 2002.2 Disponível em: <https://avmajournals.avma.org/doi/abs/10.2460/javma.2002.220.994>. doi: doi.org/10.2460/javma.2002.220.994.

BUFFINGTON, T. C. A. Idiopathic cystitis in domestic cats-beyond the lower urinary tract. Journal Veterinary Internal Medicine, v. 25, n 4, p. 784-796, 2011. Disponível em: <https://onlinelibrary.wiley.com/doi/full/10.1111/j.1939-1676.2011.0732.x>. doi: doi.org/10.1111/j.1939-1676.2011.0732.x.

BUFFINGTON, T. C. A.; WESTROPP, J. L.; CHEW, D. J. From FUS to pandora syndrome: where are we, how did we get here, and where to now? Journal of Feline Medicine and Surgery, v. 16, p. 385-394, 2014. Disponível em: <http://journals.sagepub.com/doi/pdf/10.1177/1098612X14530212>. doi: $10.1177 / 1098612 \times 14530212$. 
BUFFINGTON, T. C. A.; WESTROPP, J. L.; CHEW, D. J.; BOLUS, R. R. Risk factors associated with clinical signs of lower urinary tract disease in indoor-housed cats. Journal of the American Veterinary Medical Association, v. 228, n. 5, p.722-725, 2006. Disponível

em: <https://avmajournals.avma.org/doi/abs/10.2460/javma.228.5.722>. doi: doi.org/10.2460/javma.228.5.722.

BUFFINGTON, T. C. A.; WESTROPP, J. L.; CHEW, D. J.; BOLUS, R. R. Clinical evaluation of multimodal environmental modification (MEMO) in the management of cats with idiopathic cystitis. Journal of Feline Medicine and Sugery, v.8, n.4, p. 261-268, 2006. Disponível em: < http://journals.sagepub.com/doi/abs/10.1016/j.jfms.2006.02.002>. doi: doi.org/10.1016/j.jfms.2006.02.002.

CERVIGNI, M. Interstitial cystitis/bladder pain syndrome and glycosaminoglycans replacement therapy. Translational Andrology and Urology, v. 4, n. 6, p. 638-642, 2015. Disponível em: <https://www.ncbi.nlm.nih.gov/pmc/articles/PMC4708541/>. doi: 10.3978/j.issn.2223-4683.2015.11.04.

COOPER, E. S. Controversies in the management of feline urethral obstruction.

Journal of Veterinary Emergency and Critical Care, v. 25, n. 1, p. 130-137, 2015. Disponível em: < https://onlinelibrary.wiley.com/doi/full/10.1111/vec.12278>. doi: org/10.1111/vec.12278.

DAUDON, M.; LETAVERNIER, E.; FROCHOT, V.; HAYMANN, J. P.; BAZIN, D.; JUNGERS, P. Respective influence of calcium and oxalate urine concentration on the formation of calcium oxalate monohydrate or dihydrate crystals. Comptes Rendus Chimie, v. 19, n. 11-12, p. 1504-1513, 2016. Disponível em: <https://www.sciencedirect.com/science/article/pii/S1631074816302363>. doi: https://doi.org/10.1016/j.crci.2016.08.009.

DEFAUW, P. A.; MAELE, I. V.; DUCHATEAU, L. Risk factors and clinical presentation of cats with feline idiopathic cystitis. Journal of Feline Medicine and Surgery, v. 13, n.12, p. 967-975, 2011. Disponível em: <http://journals.sagepub.com/doi/abs/10.1016/j.jfms.2011.08.001>. doi: org/10.1016/j.jfms.2011.08.001.

DORSCH, R.; ZELLNER, F.; SCHULZ, B.; SAUTER-LOUIS, C.; HARTMANN, K. Evaluation of meloxicam for the treatment of obstructive feline idiopathic cystitis. Journal of Feline Medicine and Surgery, v. 18, n 11, p. 925-933, 2016. Disponível em: <http://journals.sagepub.com/doi/abs/10.1177/1098612X15621603>. doi: org/10.1177/1098612X15621603.

DULANEY, D. R.; HOPFENSPERGER, M.; MALINOWSK, R.; HAUPTMAN, J.; KRUGER, J. M.; Quantification of Urine Elimination Behaviors in Cats with a Video Recording System. Journal of Veterinary Internal Medicine, v. 31, n. 2, p. 486-491, 2017. Dispinível em: <https://onlinelibrary.wiley.com/doi/full/10.1111/jvim.14680>. doi: https://doi.org/10.1111/jvim.14680. 
FENELON, M.; QUINQUE E.; ARRIVE, E.; CASTROS, S.; FRICAIN, J. C. Painrelieving effects of clonazepam and amitriptyline in burning mouth syndrome: a retrospective study. Internacional. Internacional Journal Oral e Maxillofacial Surgery, v. 46, n. 11, p. 1505-1511, 2017. Disponível em: <http://www.ijoms.com/article/S0901-5027(17)31404-2/abstract>. doi: 10.1016/j.jom.2017.03.032.

FERREIRA, G. S.; CARVALHO, M. B.; AVANTE, M. B. Características epidemiológicas, clínicas e laboratoriais de gatos com sinais de doença do trato urinário inferior. Archives of Veterinary Science, v. 19, n.4, p. 42-50, 2014. Disponível em: <http://revistas.ufpr.br/veterinary/article/view/35881/23599>. doi: http://dx.doi.org/10.5380/avs.v19i4.35881.

FORRESTER, S. D.; TOWELL, T. L. Feline idiopathic cystitis. Veterinary Clinics: small animal pratice. v. 45, n.4, p. 783-806, 2015. Disponível em: < http://www.vetsmall.theclinics.com/article/S0195-5616(15)00029-7/abstract>. doi: 10.1016/j.cvsm.2015.02.007.

GNANANDARAJAH, J. S.; LULICH, J. P.; ALBASAN, H.; OSBORN, C. A. Urólitos de Purina. In: August, J. R. Medicina Interna de Felinos. Rio de Janeiro: Elsevier, 6 ed. 2011. Cap. 50, p. $501-510$.

GUNN-MOORE, D. A.; CAMERON, M. E. A pilot study using synthetic feline facial pheromone for the management of feline idiopathic cystitis. Journal of Feline na Surgery, v. 6, p. 133-138, 2004. Disponível em: < http://journals.sagepub.com/doi/pdf/10.1016/j.jfms.2004.01.006>. doi: 10.1016/j.jfms.2004.01.006.

GUY, N. C.; HOPSON, M.; VANDERSTICHEL, R. Litterbox size preference in domestic cats (Felis catus). Journal of Behavior: Clinical Applcation na Research, v. 9, n. 2, p. 78-82, 2014. Disponível em: <https://www.sciencedirect.com/science/article/pii/S1558787814000033>. doi: https://doi.org/10.1016/j.jveb.2013.11.001.

HAAFTEN, K. A.; FORSYTHE, L. R. E.; STELOW, E. A.; BAIN, M. J. Effects of a single preappointment dose of gabapentin on signs of stress in cats during transportation and veterinary examination. Journal of the American Veterinary Medical Association. v. 251, n. 10, p. 1175-1181, 2017. Disponível em: <https://avmajournals.avma.org/doi/abs/10.2460/javma.251.10.1175>.

doi: org/10.2460/javma.251.10.1175.

HAUSER, P.J.; VANGORDON, S. B.; SEAVEY, J.; SOFINOWSKI, T. M.; RAMADAN, M.; ABDULLAH, S.; BUFFINGTON, T. C. A.; HURST, E. R. Abnormalities in expression of structural, barrier and differentiation related proteins, and chondroitin sulfate in feline and human interstitial cystitis. The Journal of Urology, v. 194, n. 2, p. 571-577, 2015. Disponível em: <http://www.jurology.com/article/S00225347(15)00180-9/pdf>. doi: https://doi.org/10.1016/j.juro.2015.01.090.

HEIDENBERGER, E. Housing conditions and behavioural problems of indoor cats as assessed by their owners. Appied Animal Behaviour Science, v. 52, n.3-4, p. 345- 
364, 1997. Disponível em: <http://www.appliedanimalbehaviour.com/article/S01681591(96)01134-3/abstract>. doi: org/10.1016/S0168-1591(96)01134-3.

HOSTUTLER, R. A.; CHEW, D. J.; DIBARTOLA, P. S. Recent concepts in feline lower urinary tract disease. Veterinary Clinics Small Animal Pratic, n. 35, p. 147170, 2005. Disponível em: <http://www.vetsmall.theclinics.com/article/S01955616(04)00118-4/pdf >. doi: doi.org/10.1016/j.cvsm.2004.08.006.

JONES, B. R.; SANSON, R. L.; MORRIS, R. S. Elucidating the risk factors of feline lower urinary tract disease. New Zealand Veterinary Journal, v. 45, n. 3, 1997. Disponível em: <https://www.tandfonline.com/doi/abs/10.1080/00480169.1997.36003>. doi: org/10.1080/00480169.1997.36003.

JUNIOR, A. R.; CAMOZZI, R. B. Doença do Trato Urinário Inferior dos Felinos/Cistite Intersticial. In: JERICÓ, M. M.; NETO, J. P. A.; KOGICA, M. M. Tratado de Medicina Interna de Cães e Gatos. Rio de Janeiro: Rocca, 1 ed. 2015. Cap. 167, p. 1483 1492.

KRAIJAR, M.; FINK-GREMMELS, J.; NICKEL, R. F. The short-term clinical efficacy of amitriptyline in the management of idiopathic feline lower urinary tract disease: a controlled clinical study. Journal of Feline Medicine and Surgery, v. 5, p. 191-196, 2003. Disponível em: < http:/journals.sagepub.com/doi/pdf/10.1016/S1098612X(03)00004-4 >. doi: 10.1016/S1098-612X(03)00004-4.

KRUGER, J. M.; LULICH, J. P.; MACLEAY, J.; MERRILLS, J.; PAETAU-ROBINSON, I.; BREJDA, J.; OSBORN, A. C. Comparison of foods with differing nutritional profiles for long-term management of acute nonobstructive idiopathic cystitis in cats. Journal of the American Veterinary Medical Association, v. 247, n. 5, p. 508-517, 2015. Disponível em: < https://avmajournals.avma.org/doi/full/10.2460/javma.247.5.508 >. doi: org/10.2460/javma.247.5.508.

LEKCHAROENSUK, C.; LULICH, J. P.; OSBORNE, C. A.; KOEHLER, L. A.; URLICH, L. K.; CARPENTER, K. A.; SWANSON, L. L. Association between patientrelated factors and risk of calcium oxalate and magnesium ammonium phosphate urolithiasis in cats. Journal of the American Veterinary Medical Association, v. 217, n. 4, p. 520-525, 2000. Disponível em: <https://avmajournals.avma.org/doi/abs/10.2460/javma.2000.217.520>. doi: doi.org/10.2460/javma.2000.217.520.

LEKCHAROENSUK, C.; OSBORN, C. A.; LULICH, J. P. Epidemiologic study of risk factors for lower urinary tract diseases in cats. Journal of the American Veterinary Medical Association, v. 218, n. 9, p. 1429- 1435, 2001. Disponível em: <https://avmajournals.avma.org/doi/abs/10.2460/javma.2001.218.1429>. doi: org/10.2460/javma.2001.218.1429.

LITTLE, S. E. Trato Urinário Inferior. In: LITTLE. S. E. O Gato - Medicina Interna. Rio de Janeiro: Rocca, 1 ed. 2016. Cap. 4, p. $944-975$. 
OSBORN, C. A.; LULICH, P. J.; TCHUMCHAI, R.; ULRICH, L. K.; KOEHLER, L.A.; BIRD, K. A.; BATGES, J. W. Feline urolithiasis: etiology and pathophysiology. Veterinary Clinics: Small Animal Pratic, v. 26, n 2, p. 217-232, 1996. Disponível em: <http://www.vetsmall.theclinics.com/article/S0195-5616(96)50204-4/abstract>.

MIREAUX, M.; VILLAVERDE, C.; HERVERA, M.; ROURA, X.; CAUSSÉ, E.; FERGUIER, A.; BIONGE, V.; MOUGEOT, I. Revue Vétérinaire Clinique, v.49, p. 23-29, $2014 . \quad$ Disponível em: https://www.sciencedirect.com/science/article/pii/S2214567213000860. doi: https://doi.org/10.1016/j.anicom.2013.12.001.

NERI, A. M; MACHADO, L. H, A.; OKAMOTO, P. T. C. G.; FILIPPI, M. G.; TAKAHIRA, R. K.; MELCHERT, A.; LOURENÇO, M. L. G. Routine screening examinations in attendance of cats with obstructive lower urinary tract disease. Topics in Companion Animal Medicine, v. 31, n 4, p. 140-145, 2016. Disponível em: <http://www.companimalmed.com/article/S1938-9736(16)30047-2/fulltext>. doi: https://doi.org/10.1053/j.tcam.2016.10.006.

OSBORNE, C. A.; LULICH, J. P.; THUMCHAI, R.; BARTGES, J. W.; SANDERSON, S. L.; ULRICH, L. K.; KOEHLER, L. A.; BIRD, K. A.; SWANSON L. L. Diagnosis, Medical Treatment, and Prognosis of Feline Urolithiasis. Veterinary Clinics: Small Animal Pratice, v. 26, n. 3, p. 589-627, 1996. Disponível em: <http://www.vetsmall.theclinics.com/article/S0195-5616(96)50087-2/pdf>. doi: https://doi.org/10.1016/S0195-5616(96)50087-2.

ROBERTSON, E. Feline cystitis: a case presenting with LUTS in a young female cat. UK Vet Companion Animal, v. 19, $\mathrm{n}$ 6, 2014. Disponível em: <https://www.magonlinelibrary.com/doi/abs/10.12968/coan.2014.19.6.284>. doi: doi.org/10.12968/coan.2014.19.6.284.

SILVA, B. P. L.; KNACKFUSS, F. B.; LABARTHE, N.; ALMEIDA, F. M. Effect of a synthetic analogue of the feline facial pheromone on salivary cortisol levels in the domestic cat. Pesquisa Veterinária Brasileira, v. 37, n. 3, p. 287-290, 2017. Disponível em: <http://www.scielo.br/scielo.php?pid=S0100736X2017000300287\&script=sci_arttext>. doi: doi.org/10.1590/s0100$736 \times 2017000300013$.

WAKI, M. F.; KOGICA, M. M. Urolitíase em Cães e Gatos. In: Jericó, M. M.; NETO, J. P. A.; KOGICA, M. M. Tratado de Medicina Interna de Cães e Gatos. Rio de Janeiro: Roca, 1 ed. 2015. Cap.165, p. 1462-1482.

WESTROPP, J. L.; BUFFINGTON, T. C. A. Feline idiopathic cystitis: current understanding of pathophysiology and management. Veterinary Clinics: Small Animal Pratics, n. 34, v. 4, p. 1043-1055, 2004. Disponível em: <http://www.vetsmall.theclinics.com/article/S0195-5616(04)00030-0/pdf>. doi: 10.1016/j.cvsm.2004.03.002. 